\title{
Steps to unlocking ecosystem based fisheries management: Towards displaying the $\mathrm{N}$-dimensional potato
}

\author{
This is a peer-reviewed and self-achieved version and could contain slight errors and inaccurate formatting. A journal version of the \\ paper is available: Fisheries Research Volume 209, January 2019, Pages 117-128; https://doi.org/10.1016/j.fishres.2018.07.023 \\ John G. Pope ${ }^{a_{*}}$, Troels Jacob Hegland ${ }^{b}$, Marta Ballesterosc; Kåre Nolde Nielsen ${ }^{\text {; }}$; Mika Rahikainen ${ }^{e}$ \\ a NRC (Europe) Ltd, The Old Rectory, Staithe Road, Burgh St Peter, Beccles, Suffolk, NR340BT, UK; *popejg@aol.com \\ b Innovative Fisheries Management, Department of Planning, Aalborg University, Rendsburggade 14, DK-9000 Aalborg, \\ Denmark \\ c Centro Tecnológico del Mar-Fundación CETMAR, Eduardo Cabello s/n, 36208 Vigo, Spain \\ d Faculty of Bioscience, Fisheries and Economics, UiT - - The Artic University of Norway, Troms $\varnothing$, Norway \\ e University of Helsinki, Fisheries and Environmental Management Group, Viikinkaari 2, 00014 University of Helsinki, \\ Finland \\ *Corresponding author.
}

\begin{abstract}
Any ecosystem based fisheries management system is necessarily faced with the problem of multiple objectives that trade-off against one another. Typically, objectives such as the maximization of yield, employment or profit or minimizing environmental impacts will be optimized in different parts of the decision space, which is formed of the fishing mortality rates that can be applied to the various species, given the constraints imposed by the mixed species nature of many fishing fleets. Since objectives cannot be simultaneously achieved, managers need to consider how such objectives tradeoff against one another in order to choose a balanced strategy. Normally, they also have to consider the views of different groupings of stakeholders, who often favour widely different and conflicting objectives. This is particularly difficult if stakeholders are reluctant to expose their negotiating positions. This article explores two possible approaches to developing a Decision Support Framework for the North Sea. The first is a classic Multi- Criteria Analysis (MCA) approach that was developed in cooperation with North Sea stakeholders. The implementation went smoothly for the definition of suitable scenarios, decision trees and criteria, but failed in facilitating consensus on how to set priorities at the stakeholder level. However, it remains a possible approach for higher level management to adopt. Consequently, to aid effective decision-making a simpler approach was designed to visualise stakeholders concerns both to themselves and to the managers in charge of actual decision-making. Rather than trying to achieve some joint optima of the objectives that stakeholders wish to achieve this approach seeks to avoid the solutions various stakeholder groups resent the most. This ' $\mathrm{N}$ dimensional potato approach' proposed here treats the decision space as analogous to a partially rotten potato that has to be prepared for the table: each group of stakeholders cut away those parts of the decision space that they consider unacceptable. Ideally, this would leave a decision space where somewhat acceptable compromise solutions exist. But, if no decision space is left after all have made their cuts, this approach will still inform managers about the consequences of different solutions in terms of which group will be disappointed and by how much. Making this approach operational requires both uncovering various stakeholders' views of the unacceptable areas, and also displaying these areas in a convenient fashion together with areas of stakeholder consent. The article describes the steps taken to address these two tasks by the North Sea case study of the MareFrame research project.
\end{abstract}

Keywords: EBFM; Decision Support; Stakeholder views; Multi-attribute utility; North Sea

\section{Highlights}

- This paper presents the evolution of the decision support framework for the North Sea during the MAREFRAME project. This evolution was from a multi-criteria Analysis (MCA) approach to the N Dimensional Potato Approach. - It presents an initial classification of the stakeholder subgroups and quantifies unacceptable levels of the major concerns.

- It shows how the conflicting views of these stakeholder subgroups can be communicated clearly to senior managers 


\section{Introduction}

The European Union (EU) has committed itself to implement an ecosystem based fisheries management (EBFM) approach (Dolan et al., 2016; Link and Browman, 2014; Ramírez-Monsalve et al., 2016a,b) to be applied (EC, 2013). Overall progress has been achieved for example through the introduction of multi-annual, multispecies fisheries management plans or through a better articulation of the environmental and social dimensions in the fisheries policy although in a rather piecemeal basis. A central problem in pursuing EBFM is the need for the fisheries management system to strike a balance between diverse objectives. This is difficult because EBFM must ultimately address the interactions of fishing on all ecosystem components as well as how the various fisheries best use the system. In fisheries systems human impact is typically applied by a wide range of fishing fleets that target and produce by-catch on different species and these species will often interact through predation processes.

The task is rendered even more difficult because in applying EBFM managers also need to consider the views of diverse groups of stakeholders who characteristically have widely different and conflicting objectives. Thus, the favoured objective of a particular group of fishers might relate to yield maximization while others favour maximizing employment or profit. Moreover, even if different groups of fishers favour the same type of objective they may still be in conflict as to how it should be pursued for different fleets that fish different species. Meanwhile environmental NGOs will be concerned with reducing or avoiding environmental impacts. Such diverse objectives will necessarily be optimized in different parts of the decision space formed of either the fishing mortality rates that can be applied to the various species or to the fishing effort of the different fishing fleets. The complexities involved rule out simpler decision making approaches such as the traditional nostrum of managing each fish stock to achieve its single species maximum sustainable yield (MSY). This is in part because maximum yield for all species is not jointly feasible in an interactive species system (ICES, 2013) nor typically in a mixed catch system (ICES, 2017). Moreover, an MSY approach does not address the real objectives of fishing such as profit or employment even for single species management (Larkin, 1977).

In response to the limited advances in implementing EBFM, in 2014 the EU funded a large research project (MareFrame) to remove the barriers that prevent a more widespread use of the EBFM in Europe. The MareFrame project developed decision support frameworks for seven case studies covering all the EU sea basins. Each case study involved a scoping exercise and the use of the outputs of ecosystem-models to support structured decision-making (using Bayesian Influence Diagrams or multicriteria analysis, MCA) in an iterative stakeholder engagement process.

MareFrame deployed a co-creation approach to generate knowledge that has scientific acceptability (credibility), policy relevance (salience) and social robustness (legitimacy) (See Ballesteros et al., 2018). This approach is established as being relevant for transdisciplinary and problem oriented research. Tress et al. (2004), define transdisciplinary research "...as projects that involve academic researchers from different unrelated disciplines as well as non-academic participants, such as land managers, user groups and the general public, to create new knowledge and theory and research a common question". Facing similar challenges, an equivalent definition of transdisciplinary research is as pertinent to EBFM as it is in a landscape ecological context.

Essentially, the co-creation approach ensured that stakeholders were involved in the entire research process, which following Mauser et al. (2013) may be divided into the three stages of co-design, co-production and (co-) dissemination of results, in a continuous and iterative process to improve outcomes. The co-design phase involved cooperating with stakeholders to frame the problem focus, and to outline the general research approach to be deployed, while considering the practical constraints given by the project tasks committed to, and the availability of expertise, time and resources. Existing policy objectives (notably a requirement to satisfy Maximum Sustainable Yield (MSY) targets in terms of $F$ and SSB for commercial fish stocks) represented another important constraint, as a failure to take such objectives into account would undermine the relevance of the research. Policy relevance is one of the aforementioned criteria of the co-creation approach. In the North Sea case study codesign was first addressed at a meeting held in London in May 2014 with a wide range of stakeholders who were asked to bring their most pressing problems to the table and to prioritize them. It was further focused by discussing the evolving solutions with them at their own meetings. In the co-production phase, the project researchers had the main responsibility of performing the main scientific work related to developing a decision support framework of relevance for the identified problem focus. The involvement and impact of stakeholders was less intense in this phase compared to the previous stage but was nevertheless important to enhance the quality of preliminary outcomes and to provide feed-back on prototypes of the decision support tools to allow necessary adaptations. The final dissemination phase involved stakeholders testing the developed decision support tools and providing feedback about their utility and potential for further development and use. Co-creation has proven to lead to benefits beyond what could be achieved through traditional research (Ballesteros et al., 2018) channelling scientific findings as usable and accessible for decision-makers to support adaptive fisheries governance (see Cvitanovic et al., 2015).

The North Sea fisheries system is a dominant component for the EU's EBFM implementation, with many fish species, many different fishing fleets and multi-level governance settings. For the North Sea, the most relevant stakeholders (various groups of fisheries interests and environmental NGOs) were involved by including as project partners the two EU advisory councils whose mandates include the North Sea: the North Sea Advisory Council (NSAC) and the Pelagic Advisory Council (PELAC). The North Sea is managed jointly by the EU and Norway; since the project is focused in the EU policy context, stakeholders from third countries operating in the area were not involved in the work presented here.

The task to be addressed was to provide a decision support framework for a North Sea EBFM. The MAREFRAME agreed approach to providing decision support frameworks for all areas was to use either multi-criteria analysis (MCA) or where possible Bayesian Influence Diagrams (BID) to gain understanding about their merits and weaknesses in participatory modelling. So for consistency the initial approach to a North Sea decision support framework was to develop a MCA for the area. This decision to use MCA was taken at a MAREFRAME wide workshop rather than in consultation with stakeholders but they were closely involved in the subsequent implementation. MCA (Janssen, 2001; Kowalski et al., 2009; Sheppard and Meitner, 2005) jointly considers the utility relating to criteria, which in turn are associated with objectives such as value, profit, job creation, catch or good environmental status (GES) outcomes of the system. In the context of EBFM, this approach presupposes that well-established model(s) can predict the consequences of different management decisions. Given such models, MCA provides one possible though discrete solution: the model(s) estimate the outcomes of various alternative management scenarios, and then - through the MCA - the various objectives are weighted so that the 'best' scenario (optimal 
trade-offs) can be identified. This is a viable approach in situations where decision-makers agree on the overall problem structure, on the relevant objectives when evaluating alternatives, and on the shape of the utility functions for each indicator. Clearly the outcomes of an evaluation with an MCA will be more acceptable to the involved decision-makers if they agree broadly on how the different criteria are weighted.

The Minimum Sustainable Whinge of Pope (1983) provides an alternative but potentially complementary approach to decision support to MCA. This approach identifies those parts of the decision space that avoid regions where any stakeholder grouping would

be incensed. Thus, the strategy is not to seek for the optima but to avoid the multiple pessima of the system as seen by the different stakeholders. This area of possible compromise between stakeholders is the so-called "minimum sustainable whinge region". However

Pope (1983), for the sake of simplicity, restricted the criteria to long-term profit, jobs, and catch for a very simple single species system, and he considered only the opinions of stakeholders with pure strategies of profit, employment, catch or restricting fishing mortality.

This allowed the problem to be depicted in two dimensions as a triangular diagram. Hilborn (2010) extended this approach into the idea of pretty good yield but still for a single species system.

These approaches have the virtue that they address the reality that political practicalities may often require managers to have a certain amount of wriggle room in their decisions. In practice decision-makers often, like a young St Augustin, have to pray: " $D a$ mihi castitatem et continentiam, sed noli modo"1 since often they have to choose politically expedient solutions rather than some potentially more ideal solution.

Such an approach -- to avoid anyone's glass 'being half empty' rather than only one glass being full2 -- can be extended to EBFM thinking. However, it will clearly need a different presentation compared to the simple two dimensional approach of Pope (1983) to communicate its results to stakeholders and the relevant decision-makers. This follows from the fact that the information to be communicated are the far more complex management criteria values and the various stakeholder positions that are to be expected in a multispecies, multi-fleet EBFM system. This seems more difficult for stakeholders or senior managers to visualise.

\subsection{The N-dimensional potato approach}

Fortunately, a clear way to visualise this process was described by a stakeholder (Michael Andersen, pers. comm.) as analogous to the idea of preparing an ' $\mathrm{N}$-dimensional potato' for cooking. When preparing a real potato for cooking you need first to cut off unpalatable parts. Such is the concept of the $\mathrm{N}$ dimensional potato display; the model provides the ' $\mathrm{N}$ dimensional potato' (the possible criteria outcomes at all values of fishing mortality of the decision space) and any parts of this decision space that are unpalatable to any stakeholder group need to be cut off if a palatable compromise solution is to be found. Thus we have to describe what criteria outcomes, estimated by the model(s), are considered unpalatable from the point of view of the different groups of stakeholders and need to be cut off - and then ideally management can focus on what remains (if anything is left).

The N Dimensional Potato concept seems to have an intuitive appeal to stakeholder that the Minimum Sustainable Whinge lacked. Hence this name is adopted in this paper. However, to turn it from a concept into a viable Decision Support Approach requires two problems to be solved:

- Firstly, how to uncover stakeholders' perceptions of what are the unpalatable parts from each of their perspectives? - Secondly, how to display the stakeholders' perceptions in a convenient fashion that informs decision-makers of areas of stakeholder consent? Or, if no area of consent exists, how to indicate which stakeholder grouping will not consent to a given solution?

Hence, the objective of this paper is to describe the steps taken to develop a viable Decision Support Framework for supporting the implementation of an EBFM that has been tested in the North Sea. The article aims to describe and discuss the preliminary results of a process of asking stakeholders in the North Sea for their judgement of the main problems associated with EBFM and of what criteria causes them most concern (while at the same time discussing the process itself) and then describe the paths taken to developing viable Decision Support Frameworks for the implementation of the EBFM.

The article does not aim to deliver more than a brief description of the scope of the North Sea T-ONS model (Trade-Offs North Sea previously known as the Green Model). This model provides the background calculations for both the MCA and the Potato Approaches. These are thoroughly described in Pope (2018) but this article does seek to describe how it is used to visualise stakeholder perceptions.

\section{Materials and methods}

\subsection{Co-design and early development of the T-ONS model}

The case study was carried out through a structured dialogue with stakeholders (see Table 1), implementing the co-creation approach. Stakeholders included Industry and NGO representatives from PELAC and NSAC, national scientists through ICES and also managers and policy-makers, explicitly acknowledging that collaboration is the proper path to address the complex societal challenges of the North Sea. 
Table 1 Interactions with stakeholders during the MareFrame North Sea case study.

\begin{tabular}{|c|c|c|c|}
\hline Date & Type of interaction* & Participants & Place \\
\hline $04 / 02 / 2014$ & NSAC -WG & NSAC Stakeholders & London \\
\hline $14 / 05 / 2014$ & Co-creation & NSAC-\& PELAC Stakeholders & London \\
\hline $19 / 10 / 2014$ & ICES WG & ICES Working Groups WGSAM* and WGMIXED & London \\
\hline $12 / 11 / 2014$ & NSAC-WG & NSAC Stakeholders & Brussels \\
\hline $11 / 03 / 2015$ & Co-creation & PELAC & Webex \\
\hline $28 / 04 / 2015$ & Co-creation & NSAC & Webex \\
\hline $21 / 09 / 2015$ & Theme Session & ICES Annual Scientific Conference (scientific stakeholders) & Copenhagen \\
\hline $15 / 10 / 2015$ & NSAC-WG & NSAC & Hamburg \\
\hline $12 / 02 / 2016$ & Co-creation & PELAC & Webex \\
\hline $26 / 02 / 2016$ & Co-creation & PELAC & The Hague \\
\hline $17 / 03 / 2016$ & Presentation & DGMARE Lunch Time Lecture & Brussels \\
\hline $11 / 04 / 2016$ & Co-creation & PELAC & The Hague \\
\hline $13 / 07 / 2016$ & Co-creation & NSAC-PELAC & Aberdeen \\
\hline $13 / 09 / 2016$ & Theme Session & ICES ASC (scientific Stakeholders) & Riga \\
\hline $10 / 11 / 2016$ & Conference & BENA (scientific Stakeholders) & Bucharest \\
\hline $26 / 02 / 2017$ & Presentation & EU Parliament, Fisheries Committee & Brussels \\
\hline $19 / 04 / 2017$ & NSAC-WG & NSAC Stakeholders & The Hague \\
\hline $20 / 06 / 2017$ & Workshop & Decision-makers EU Member States & Brussels \\
\hline $16 / 10 / 2017$ & ICES WG & ICES WGSAM** & Copenhagen \\
\hline $19 / 10 / 2017$ & NSAC-WG & NSAC & Brussels \\
\hline
\end{tabular}

${ }^{*}$ Type of interaction: WG refers to interaction within an established working group session either in the North Sea Advisory Council or in ICES. Cocreation describes interactions according to the co-design, co-production and co-dissemination phases aforementioned. The theme session labels scientific conferences. Presentation and Conference include one-way communication with a target audience.

** WGSAM : Working Group on Multispecies Assessments methods and Working Group on Mixed Fisheries Advice.

As a starting point in the co-design stage, the NSAC and the PELAC members participated in a scoping exercise, defining the problems to be addressed at a joint meeting (May 2014):

- The need to achieve Fmsy.3

- The Landing Obligation.

- The Risks of Incompatible Regulation.

They required a case study of the North Sea that helped clarify these. Following, the co-production stage focused on the development of the T-ONS model to address the first of these concerns. This builds upon the approach of Collie et al. (2003), which was further developed progressively and iteratively involving industry and scientific consultations. It then still further modified to feed into the MareFrame Decision Support Framework. The T-ONS model is described in Pope (this issue2018) but briefly it is a model that emulates the behaviour of more complex multispecies models and joins the results of these to the fleet effort data so as to model both the species interactions and the mixed fishery constraints of the system. This enables the model to predict the long term catch and fishing fleet effort results of changing fishing mortality rate on any of the 12 main North Sea species. In turn these results are interpreted to provide those fisheries, social, economic and good environmental criteria outcomes that can presently be quantified for any given scenario of fishing mortality change. Moreover it is able to optimise specific outcomes to develop the scenarios that stakeholders wanted to consider (e.g. Maximum Sustainable Catch Value, a multispecies analogue to Maximum Sustainable Yield). Additionally T-ONS is designed to be swift in response, transportable across computers, and user friendly. Hence, it is suitable for stakeholders and senior managers alike to use on their own computers and is available on the MareFrame website.4 Stakeholders and managers can use it to develop their own additional scenarios or make subtle modifications to existing scenarios. 
The other two problems identified by stakeholders, the landing obligation and the risks of incompatible regulation had a lower priority in the MareFrame agenda that both researchers and stakeholders were committed to.5. However, progress has been made on these subjects and is reported in Pope and Weber (2018).

\subsection{Co-production: advances in the decision support framework using MCA}

The first attempt to develop a Decision Support Framework was by applying a MCA approach. Following the MareFrame research design a MCA framework was developed for the North Sea, in collaboration with representative stakeholders drawn from both NSAC and PELAC. This was achieved using qualitative (focus groups, case study meetings) and mixed quantitativequalitative $(\mathrm{MCA})$ research techniques. In cooperation with stakeholders a set of objectives and alternative management scenarios were defined.

The scenarios chosen were:

$1 \mathrm{BAU}=$ Business as Usual i.e. Status Quo Fs.

2 Max. Econ. Yield i.e. Maximum profit.

3 Max. GVA = Maximum Gross Value Added i.e. Maximum of Profit plus Labour Costs.

4 Max Value i.e. maximizing the Value of all landings.

5 Conservation i.e. Reduction in all Fs to $75 \%$.

6 All SSB above Blim Then Max Profit i.e. maximizing profit subject to the constraints that each stock's spawning biomass was above its limit reference point.

7 Max. Pelagic Profit i.e. Maximum Profit for the Pelagic Fleets.

8 Max. Dem Profit i.e. Maximum Profit for the Demersal Fleets.

The North Sea T-ONS model was used to optimise the relevant criteria for scenarios 2-4, 6-4 and 6-8. The structure of the component decision trees (Fig. 1A-C) and the overall decision tree (Fig. 1D) was successfully agreed with representative stakeholders from NSAC and PELAC. The criteria chosen by the stakeholders are shown in the 3rd or 4th levels of Fig. $1 \mathrm{~A}$ and $\mathrm{B}$ and the 2 nd level of Fig. 1C. All the criteria values for each Scenario were estimated using the T-ONS model and are available as tables in the model.

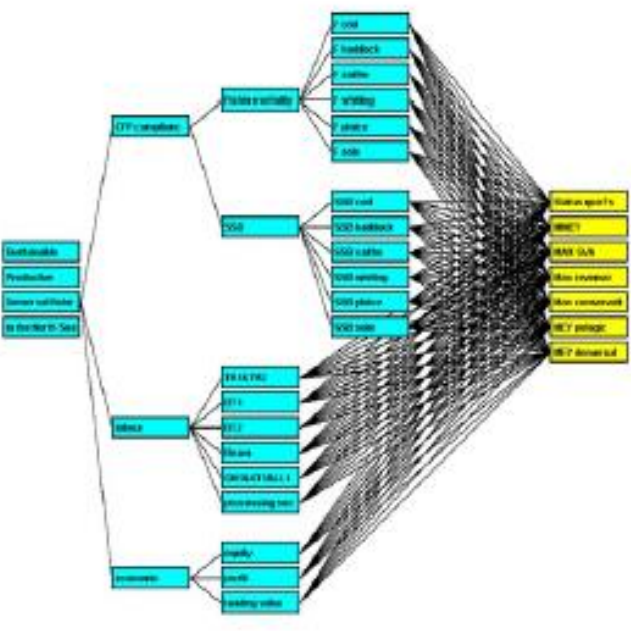

c) Decision tree for the ecosystem considerations

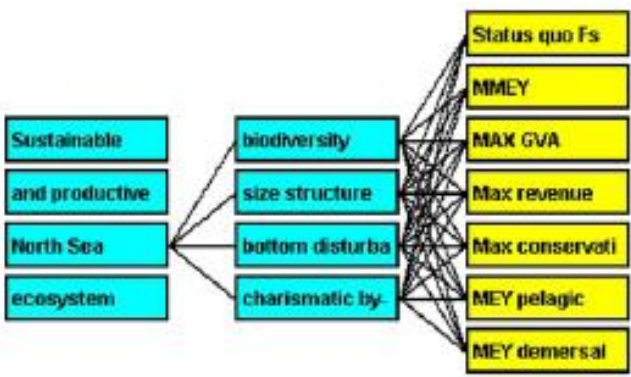

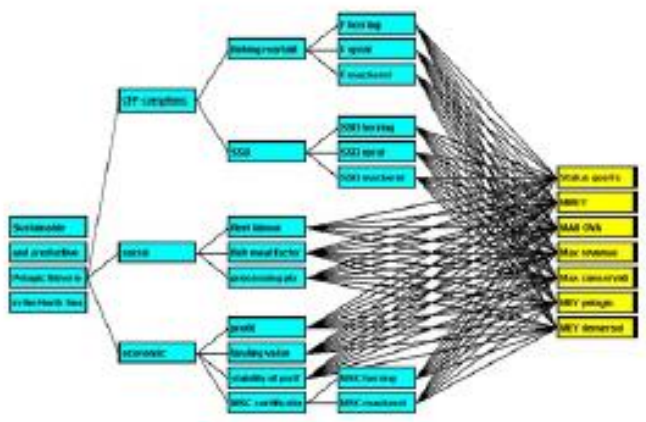

D) The synthesis Decision tree

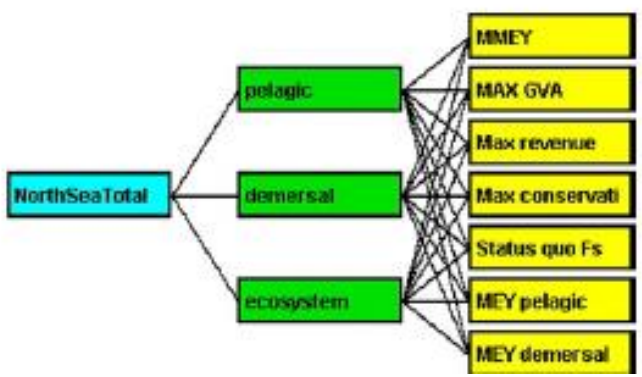

Fig. 1 The hierarchical decision tree for the North Sea EBFM decision support. The project staff co-creatively developed distinct decision trees with the NSAC (1 A) and PELAC members (1B). They describe the goals and concerns of the fisheries sector. Ecosystem concerns (1C) were divided off in a separate decision tree. These three decision trees are merged and weighted against each other in a synthesis tree (1D).

Carefully designed and well-supported attempts were made to get North Sea stakeholders' weightings for an MCA. These attempts included both on-line meetings and face-to-face workshops. Online weighting tools were developed for this purpose to help stakeholders decide on weightings. These were interactive spreadsheets that were developed to help individual stakeholders decide on weightings and to displayed their results. Fig. 2 illustrates the demersal fisheries weighting spreadsheet. 
These sheets can be made available to senior managers on request. Aggregate consensus weighting was then attempted using Web-Hipre (Mustajoki et al., 2004).

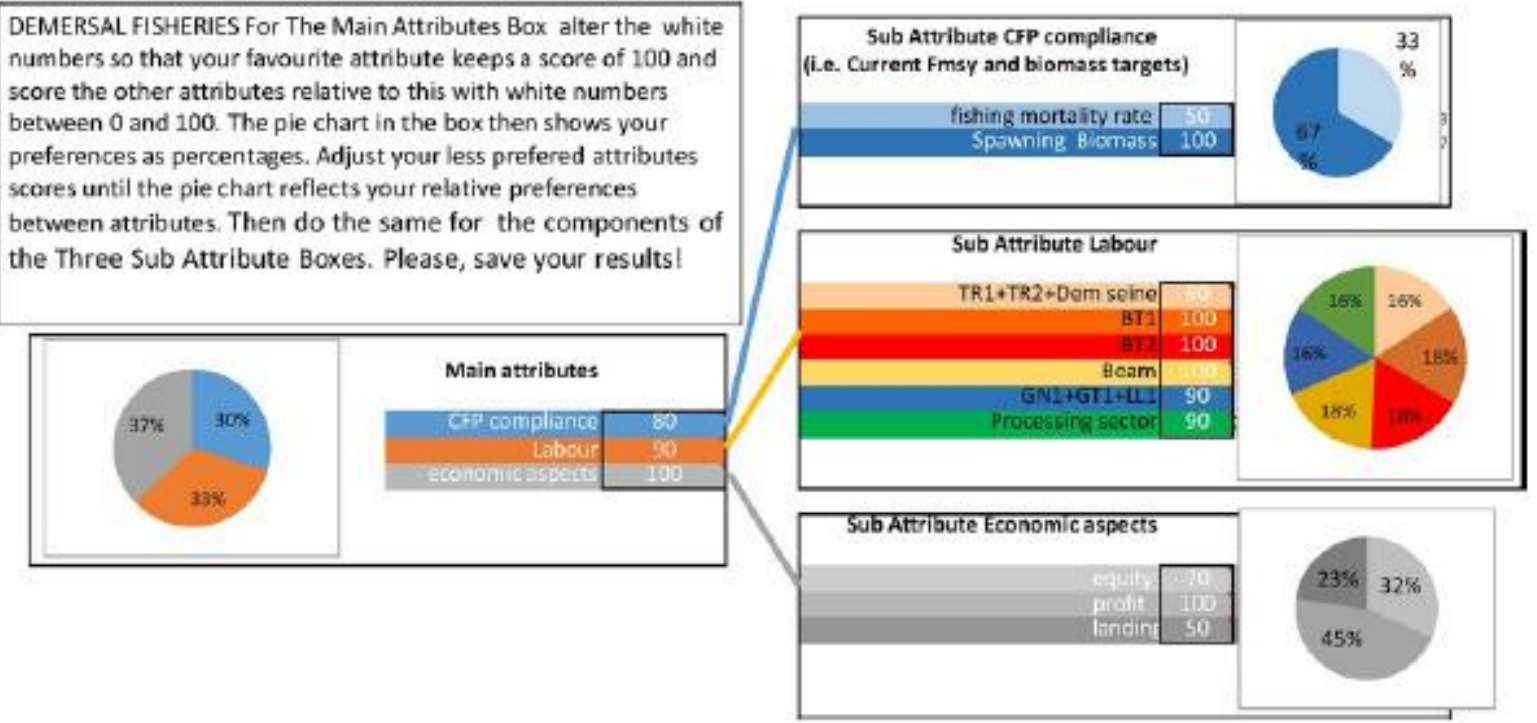

Fig. 2 The demersal fisheries weighting spreadsheet developed to help and encourage stakeholders to provide MCA weightings.

Unfortunately, the efforts to obtain agreed consensus value functions and weightings largely failed or had low response rates at the stakeholder decision level. Nevertheless, this work provided considerable insight into the range of stakeholder concerns. The many analogies between the approaches aided in the smooth transition from the MCA to the N-dimensional potato approach (Table 2).

\begin{tabular}{|l|l|l|}
\hline Logical steps & MCA & N-dimensional potato approach \\
\hline $\begin{array}{l}\text { Structuring the objectives } \\
\text { hierarchy }\end{array}$ & $\begin{array}{l}\text { a decision tree with performance indicators } \\
\text { (Fig. 1) }\end{array}$ & a vector of performance indicators (Fig. 4) \\
\hline Scoring & $\begin{array}{l}\text { continuous value functions for performance } \\
\text { indicators }\end{array}$ & $\begin{array}{l}\text { discretized zones using a TLS; red is } \\
\text { regarded a veto }\end{array}$ \\
\hline Weighting & $\begin{array}{l}\text { hierarchical weighting using e.g. SWING } \\
\text { method }\end{array}$ & $\begin{array}{l}\text { no quantitative method to express relative } \\
\text { importance of performance indicators }\end{array}$ \\
\hline $\begin{array}{l}\text { Ranking of the decision } \\
\text { alternatives }\end{array}$ & $\begin{array}{l}\text { decisions ordered based on their overall } \\
\text { value; calculated as the sum of weighted } \\
\text { scores }\end{array}$ & $\begin{array}{l}\text { heuristic selection; alternatives having many } \\
\text { red lights are not preferred }\end{array}$ \\
\hline Sensitivity analysis & available & not available \\
\hline
\end{tabular}

\subsection{Co-production: from the MCA to the N-Dimensional Potato as a decision support framework}

Given the failure to achieve consensus on how to weight different concerns in the MCA in meetings with stakeholders, attention was then focused on how to help senior decision-makers in this process. It was considered that senior decision-makers need both to clearly understand the trade-offs between decision criteria and also to fully understand the concerns of stakeholders. Moreover as noted in the introduction political practicalities may also require them to have a certain amount of wriggle room in their decisions.

To provide senior decision-makers with evidence-based support to tackle these complex problems based on the criteria of scientific robustness, policy relevance and social legitimacy, a simpler approach was required particularly with respect to communicating stakeholder preferences. This needed stakeholders to be classified into different groups associated with their main concerns and to uncover each groups perceptions of what are the unpalatable levels of their main concerns. Consequently, a pilot study questionnaire was constructed in order to create a basis for defining thresholds for a traffic light system (TLS). The questionnaire used is shown in Fig. 3. The questionnaire asked stakeholders to record their views of what three concerns of fisheries management they considered most important, and for each of these most important concerns to indicate what the unacceptable (unpalatable) and unattractive levels of the concern criteria might be. Two of our authors (JGP and TJH) presented this briefly to the Demersal Working Group of the NSAC in April 2017. Despite it being a rough draft questionnaire and stakeholders being asked to complete it in the course of a busy meeting, the response rate was encouragingly high and 16 completed questionnaires were received. Clearly, this is a very small number and does not include the PELAC (or in general stakeholders not present at this particular meeting of the NSAC's Demersal Working Group) but gave enough information to get the flavour of the problem and to suggest how the questionnaire might be improved for future use.

\footnotetext{
${ }^{\text {a }}$ This veto does not imply an effective veto over the choice of alternatives by the final decision-makers.
} 
What 3 Concerns of Fisheries Management are Most Important to You

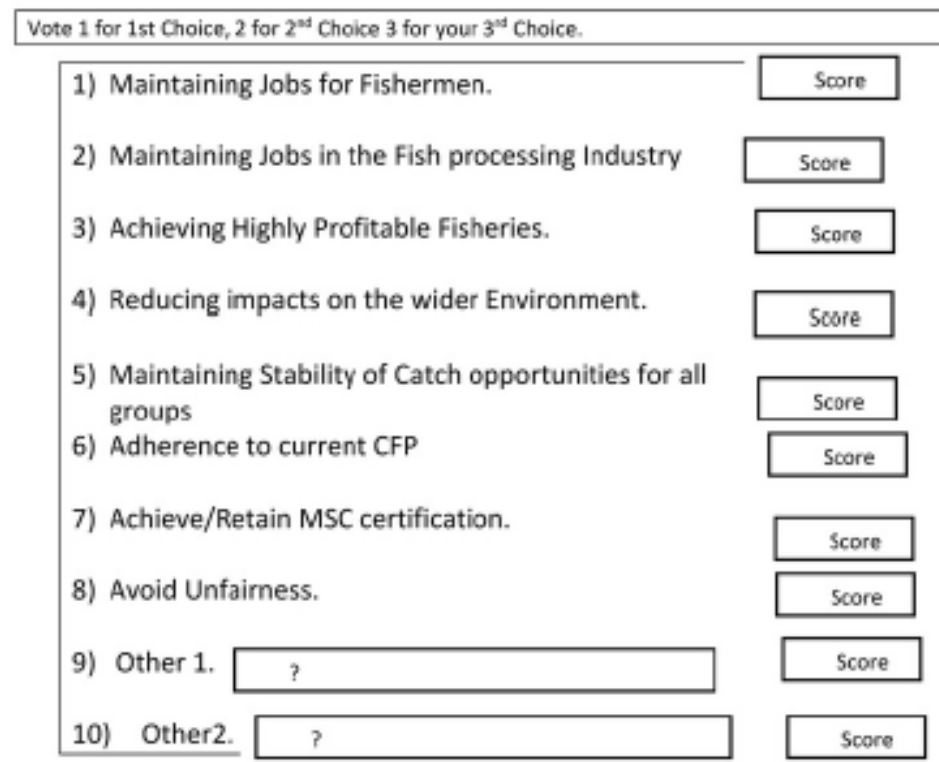

For Your 3 Most important Concerns say what would be a Rotten Outcome Where possible give bad outcomes in absolute terms e.g. Profitability less than $5 \%$ or 5 stocks under Blim.

When this is not easy relate your answer to the current situation.

e.g Emplayment less than $50 \%$ of current levels

\begin{tabular}{|c|c|c|}
\hline Concern & Rotten Level & Unattractive \\
\hline 1) & & \\
\hline 2) & & \\
\hline 3) & & \\
\hline
\end{tabular}

Fig. 3 The questionnaire used in the pilot investigations.

The small number of results currently available preclude vigorous statistical methods for classification so results were prepared in tabular form for an initial interpretation by eye. The criteria levels of what was of most concern to each sub-group were then tabulated as maximum, median and minimum for both the unacceptable (Red) levels and uncomfortable (Yellow) levels. This was to establish the likely unacceptable and unappealing level of the issues of most concern to the stakeholder sub-groups. Where responders, who belonged to the other sub-groups, gave numerical criteria values for these concerns, then these were also included as long as these appear to have been offered in the same spirit as sub-group members.

Having gathered information on the main stakeholder concern groups and the thresholds of unpalatable levels of their concerns a second challenge remained. This was how to display the stakeholders' perceptions in a convenient fashion that informs decisionmakers of areas of stakeholder consent? Or, if no area of consent exists, how to indicate which stakeholder will be offended with a given olution? To achieve this the displays of the T-ONS model were modified by including a TLS to show whether particular stakeholder groups were comfortable or unhappy with the levels of criteria of particular concern to them.

The approach taken to implement these ideas build on the concept of traffic light systems (TLSs). Foden et al (2008) briefly reviewed the main advantages and disadvantages of using TLSs in aquatic environmental assessments. They observed that the main advantages of TLSs are that they support users in observing and getting an overview of changes, and also simplify the presentation of complex data. The main drawbacks of TLSs, include the concern that the categorisation into colour scheme may be subjective, that they may lead to that detailed changes are ignored as these are summarised in a number of broad categories, and that outcomes can be influenced by the chosen number and ranges of categories. The T-ONS model seeks to mitigate the mentioned disadvantages of a pure TLS display by providing additional dashboard displays of the values of key variables. The T-ONS model which is constructed in Microsoft Excel, offers considerable flexibility of display. This allowed the MareFrame design philosophy of using co-creation to jointly develop products of value to stakeholders to proceed incrementally. Suitable displays of criteria were developed to present to a meeting of the NSAC Demersal Working Group.

Displays were further developed in preparation for a presentation to high-level fisheries managers (primarily national civil servants) at a workshop at the Directorate General for Research and Innovation in Brussels on 20thth June 2017. The workshop used a TONS like model of a fictitious case study (Atlantos), which included many features simplified from the North Sea case study. The fictitious case study was adopted both to be feasible for use in the limited time available at the workshop, to make the example interesting to a wider audience, and to render the results politically non-contentious; the main display of the case study model appears as Fig. 4. This features both the main criteria values and a TLS display of those criteria levels supposed to be of concern to the different groupings of stakeholders. 


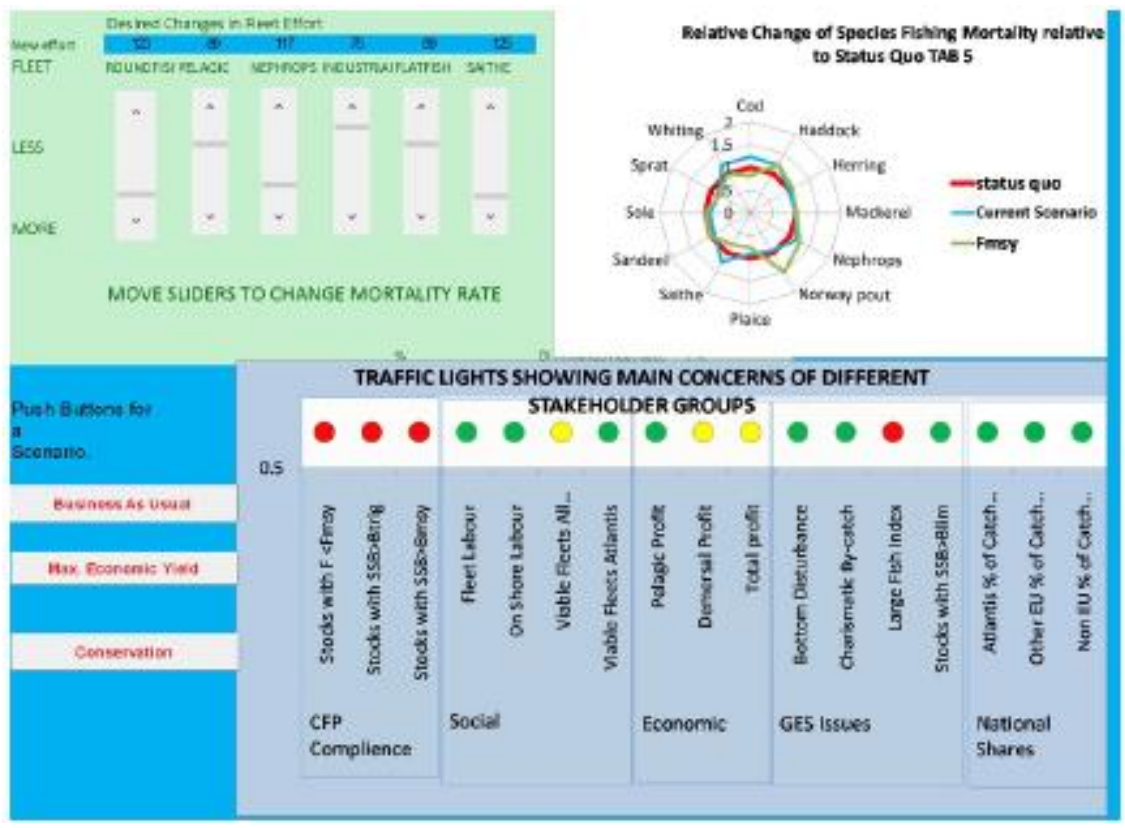

Fig. 4 Part of the Control Panel display of the Atlantos version of the T-ONS model used in the Senior Managers Workshop held in June 2017 Brussels. This shows the sliders, Scenario Buttons and the Traffic Light Display. Fishing mortality rates are set to the Maximum Economic Yield Scenario.

Workshop participants suggested several useful improvements that have been incorporated into the display of the North Sea TONS model together with the trigger levels for traffic lights provided by the North Sea stakeholders. Traffic lights are readily constructed ${ }^{6}$ in Excel.

\section{Results}

\subsection{Implementation of the co-creation approach}

The implementation of the co-creation approach (for more detail see Ballesteros et al., 2018) has provided relevant findings:

1 Stakeholders have an interest in being engaged in applied research activities, as proven by the interaction that took place during the implementation of the case study (20 events, see Table 1$)$. This interest is connected to the capability of the research outputs to generate advocacy coalitions that affect current fisheries management.

2 The co-design stage (setting priorities and defining scenarios) was carried out through a structured dialogue framed within the current policy objectives. Some of the stakeholder defined problems (landing obligation and incompatible regulation) were acute issues particularly for the sector and have been

Table $3 \mathbf{b}$ Classification of stakeholders by their 2 most important criteria of concern. Table entries are counts of times a criteria was recorded as being the first or second concern by three sub groups. Note that the jobs in processing criterion and the fairness criterion were not scored as a first or second choice criteria by any participant so they are eliminated from the table in the interests of clarity.

\begin{tabular}{|c|c|c|c|c|c|c|}
\hline \multirow{2}{*}{$\begin{array}{l}\text { Sub- } \\
\text { groupings } \\
\text { of NACC } \\
\text { Stakeholder }\end{array}$} & \multicolumn{6}{|c|}{ Criteria of Concerns } \\
\hline & Jobs at sea & Profit & Environment & Stability all fleets & $\begin{array}{l}\text { Adherence } \\
\text { to CFP }\end{array}$ & MSC Certification \\
\hline $\begin{array}{l}\text { Group } 1 \\
\text { Concerned with (Fishermen Jobs) }\end{array}$ & 5 & 2 & 0 & 1 & 0 & 1 \\
\hline $\begin{array}{l}\text { Group } 2 \\
\text { Concerned with (Profitstability) }\end{array}$ & 0 & 2 & 0 & 5 & 1 & 1 \\
\hline $\begin{array}{l}\text { Group } 3 \\
\text { Concerned with (EnvironmentCFP) }\end{array}$ & 0 & 0 & 3 & 0 & 4 & 0 \\
\hline
\end{tabular}

\subsubsection{Unacceptable (Red) levels and unattractive (Yellow) levels of criteria of concern}

Table 4 shows the maximum, median and minimum levels of the unacceptable (Red) levels and unattractive (Yellow) levels of the criteria of concern named on the questionnaire, which could be interpreted in terms of actual or potential model outputs. The results were interpreted as being relative to current criteria values. However, the initial questionnaire was not clear on the basis on which answers should be provided and some answers consequently required sympathetic interpretation.

\subsection{Model Display}

Fig. 4 shows the model display developed for the workshop with national and EU decision-makers held in June 2017 (see Table 1). This features key criteria values together with a TLS display of stakeholder preferences. It is controlled either by using the sliders for the fishing mortality rate for each species relative to status quo or by pushing one of the three scenario buttons. 
Moving sliders or pushing scenario buttons provide immediate results in terms of changes in the predicted outcome - and thereby traffic lights, which indicate where the outcome may conflict starkly with the preferences of one or more stakeholder groups.

The participants assessed the potential of the T-ONS model that includes the (TLS) "potato" display to explore and compare alternative management scenarios. It was observed that putting the decision making issue into a logical structure is useful, and helps to reveal trade-offs. For instance, this will enable the users of the T-ONS model to evaluate how a management strategy that is advocated by a particular interest group may be detrimental to the interest of another group (or the general interest). There is also an intrinsic value in communicating and explaining "tough" decisions by making trade-offs transparent, or identifying reasons for deadlock in negotiations.

Applying MCA was initially perceived by the participants as complex and "hard to get hold of"; this contrasts with the wide acceptance of the 'TLS display' embedded in the T-ONS model. Apparently, the weighting (setting preferences for subobjectives and indicators) and scoring (defining value functions for indicators) were particularly challenging.Decision makers currently apply other methods to elucidate what matters and how important is a specific topic to the different stakeholders in a given decision setting. These range from organizing meetings to generate conceptual and mutual understanding of the topic to developing impact analysis that make explicit the likely consequences of alternative choices. The dichotomy of winners/losers is enriched with the $\mathrm{N}$-dimensional potato approach, which helps decision-makers identify bargaining and compromise areas central to stakeholders stated preferences.

As potential users of the T-ONS model, the decision-makers demanded ownership of the process. For that purpose, they suggested several improvement ideas: to add an overview threshold that facilitates the understanding of what triggers the trafficlight; to explore the possibility of adding new topics (e.g. gear selectivity or area based issues as long term closure as a management measure); to reinforce that the output is easy to grasp, simplifying the analysis (e.g. explanatory pages).

\section{Discussion and conclusions \\ 4.1 Utility of the co-creation approach}

The implementation of the co-creation approach enables a structured dialogue among multiple stakeholders, supported by tools (T-ONS \& MCA and T-ONS \& N-Dimensional Potato) that graphically illustrate the likely consequences of their positions and choices. These tools were built on scientific evidence and bought transparency to the process. Sometimes, as in the case of the MCA, they require more openness than many players are willing to supply. By fostering the dialogue between evidence-based (scientists) and experience-based (industry) knowledge providers and decision-makers, the co-creation approach has delivered a research output (the $\mathrm{N}$-Dimensional Potato). Its value relies mainly in its capability for decision aiding rather than for consensus building or dispute resolution. Its strength, due to the design process, lies in the fact that usability and applicability reinforce the stakeholders' potential uptake.

\subsection{The MCA approach}

The MCA remains a potential approach for a Decision support Framework for the North Sea. However, as a method that could aid stakeholders form management proposals it failed because the stakeholders could not buy into a common weighting scheme. This impasse seemed unlikely to change and suggests, consistent with earlier research on the advisory councils (Hatchard and Gray, 2014), that the stakeholders within and between the NSAC and PELAC do not form such a cohesive set and would have difficulties themselves to adopt MCA or to propose consensual EBFM decisions. Consequently, for the North Sea the problem of applying EBFM likely devolves to more 'senior' decision-makers (managers at Member State or EU level) who themselves might be able to agree to an MCA or other approaches to the problem.

\subsection{The $\mathrm{N}$ dimensional potato approach}

\subsubsection{Classification of stakeholders and uncovering unacceptable (Red) levels and unattractive (Yellow) levels of criteria of concern}

In overview, the results from the pilot questionnaire provide a clearer idea on how to classify stakeholders into sub-groups. They also helped identify the key criteria for each subgroup of stakeholders and to specify the "unpalatable levels" of the key criteria that can be used to trigger the North Sea traffic lights. Most importantly - given that this is very much a pilot study -- the high response rate suggests that stakeholders are able to provide this data freely without feeling they are compromising their negotiating positions. Thus, it should be possible in the future to extend this method. Specifically, the questionnaire results seem to indicate three fairly separable groups of NSAC stakeholders: Those primarily concerned with fishermen's jobs, those mostly concerned with stability in the sense of preserving catch opportunities for all sectors, and those most concerned with the environment and maintaining the current CFP limit and target reference points for the fishing mortality on each stock. The study provides at least initial values for the trigger levels for changing the traffic lights used in the display. Clearly, it would be better (and ultimately necessary) to have more responses than we obtained, in particular from members of the PELAC. It is also clear in retrospect that the questionnaire should have been more specific about what numeric results were required. However, as a pilot study there were some advantages in this not being the case, since its open-ended nature threw up a number of points that we had not considered. This might in a few cases lead to new traffic lights. The written comments also give a strong feeling of important concerns that exist even if we cannot tackle them yet with the T-ONS Model.

\subsubsection{Model display}

In general stakeholders have found the TLS display a useful way of highlighting concerns. The display increases accessibility to multidisciplinary science outputs. Its relevance in terms of usability and applicability for the implementation of the EBFM in a given setting is supported by the co-design and co-production stages.

The results of the workshop in Brussels in June 2017 with senior managers (mainly national civil servants) suggested that the TLS displays given in the fictitious Atlantos (T-ONS-like) model were helpful in communicating stakeholders concerns. Adopting the lessons learned from this the T-ONS model has been adapted to reflect their comments and the results of the stakeholder survey coupled with insights gained from the MCA work with respect to the concerns of PELAC stakeholders. 
Fig. 5 shows the new TLS now used in the North Sea T-ONS model for the pre-programmed Maximum Value scenario, Business as usual (i.e. Status Quo levels of fishing) scenario and the Conservation scenario. The maximum value scenario requires the fishing mortality on all species to be increased by up to $125 \%$ of the status quo levels while the Conservation scenario requires all fishing mortalities to be decrease to $75 \%$ of their status quo levels. Thus, these three scenarios span the $\pm 25 \%$ range of fishing mortality changes allowed by the T-ONS model and it may be seen that the traffic lights change over this range but that there are always some red lights for any scenario. The traffic lights wherever possible are now calibrated to the median values given by the stakeholders in Table 4. However, the annual variation in catch concern of stakeholders has not yet been developed as a traffic light since it is somewhat beyond the scope of the present structure of the T-ONS model. Traffic lights have been included for what were considered to be the main concerns of PELAC stakeholders given their views expressed at MCA discussions.

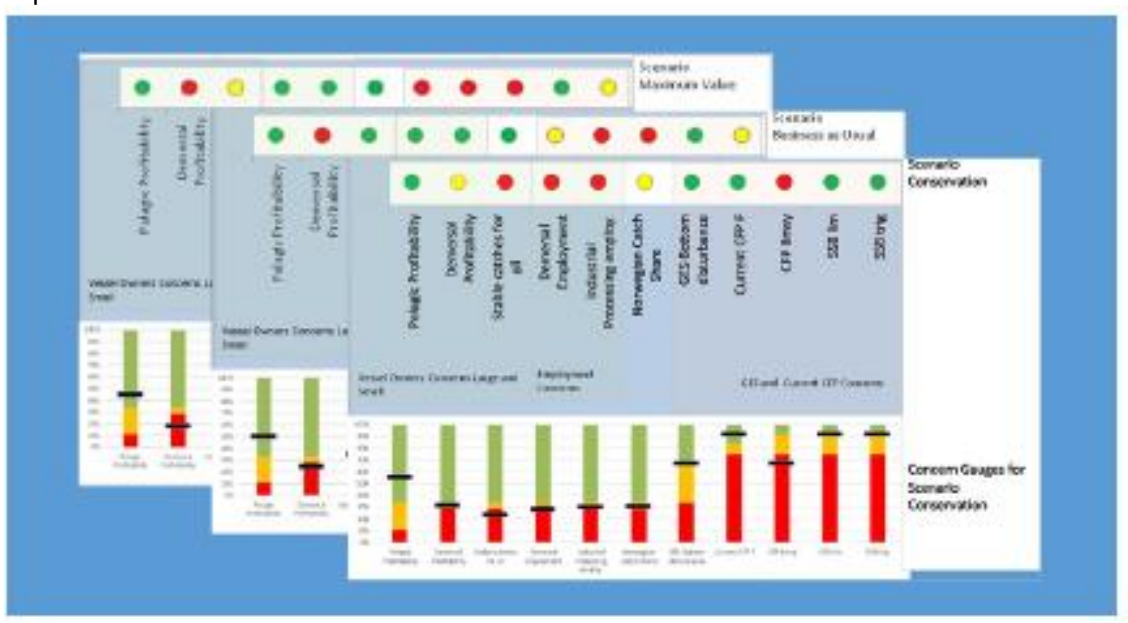

Fig. 5 Overlap Plot of the Traffic Lights for three of the eight standard T-ONS model scenarios and the associated concern gauges (only that of the Conservation Scenario being shown in full).

Table 4 Ratio of the unacceptable and unattractive levels of criteria of concern to the current baseline.

\begin{tabular}{|c|c|c|c|c|c|c|}
\hline \multirow{2}{*}{$\begin{array}{l}\text { Red } \\
\text { and Yellow Ranges }\end{array}$} & \multicolumn{6}{|l|}{ Criteria } \\
\hline & $\begin{array}{l}\text { Maintain Jobs } \\
\text { for } \\
\text { Fishermen }\end{array}$ & $\begin{array}{l}\text { Achieve } \\
\text { Profitable } \\
\text { Fisheries }\end{array}$ & $\begin{array}{l}\text { Reduce Impacts on } \\
\text { the Wider } \\
\text { Environment }^{\mathrm{a}}\end{array}$ & $\begin{array}{l}\text { Maintain Stable } \\
\text { Catches for All } \\
\text { groups }\end{array}$ & $\begin{array}{l}\text { Annual Variation } \\
\text { Of Catch }\end{array}$ & $\begin{array}{l}\text { Adherence to } \\
\text { Current } \\
\text { CFP }\end{array}$ \\
\hline Red Maximum & 100 & 90 & 100 & 80 & 50 & 100 \\
\hline Red Median & 85 & 77.5 & 100 & 80 & 50 & 75 \\
\hline Red Minimum & 70 & 50 & 100 & 50 & 50 & 50 \\
\hline Yellow Maximum & 95 & 95 & 90 & 95 & 75 & 100 \\
\hline Yellow Median & 90 & 90 & 75 & 95 & 75 & 95 \\
\hline Yellow Minimum & 85 & 50 & 60 & 75 & 75 & 70 \\
\hline Number of Quantifiable Responses & 4 & 5 & 3 & 3 & 1 & 4 \\
\hline
\end{tabular}

Note that the values on MSC certification (2 returns that were essentially comments) and fairness (no answers) are not included in the table. Moreover, the table could not include all responses since some were in comment form while others, although numeric, were clearly made on a different basis to the majority. Particularly those written into the other 1 and other 2 boxes (questionnaire boxes 9 and 10), which often express heartfelt concerns, such as a distress over the administrative burden felt by fishermen and the need to consider predator-prey relationships in fisheries management.

a Note that to satisfy environmental concerns requires lower impacts so for this criteria low is good and high bad. For all other criteria, high is good and low bad.

Fig. 5 also shows (in whole for the conservation scenario and in part for the other two scenarios) the new "Concern Gauges" that were developed in response to the workshop with senior managers, who asked the research team "to add an overview threshold that facilitates the understanding of what triggers the traffic-light". The resulting concern gauges thus form a good illustration of the power of co-creation in action. Both the TLS and the concern gauges respond not just to the preprogrammed scenarios but to any scenario a manager or stakeholder choses to input themselves.

The approach taken to implement these ideas builds on the concept of traffic light systems (TLS). In fisheries management the concepts of target reference points (desirable levels), and limit reference points (levels to avoid exceeding) (Caddy and Mahon, $1995)$ are familiar. So too is the concept of an early warning reference point (at a level set to define a level that reduces the risk of a target or a limit reference point being trespassed (Garcia, 1995). In fisheries management these three reference points are analogous to the green, yellow, and red (TLS) (Caddy, 1998, 2002; Hall and Mainprize, 2004).

As noted by Foden et al. (2008) TLS have disadvantages as well as advantages. When used for fisheries decision support the indicators need to be relevant for the problem, specific for the context, measurable, and understandable for the stakeholders. Critical to a TLS is defining the thresholds at which the lights change colour. This logic has to be understandable to decision makers to avoid a non-transparent black-box case, as evidenced during the Workshop. Moreover, a TLS dashboard may be too complex and provide overwhelming information. Aside from the amount of information, the level of complexity that works on a dashboard depends entirely on the expertise of those who use it. A practical problem is that it is likely that there will always be several red alerts on a dashboard, and users will need to decide which are most important. For this task MCA does provide a more systematic approach to balance the trade-offs between different objectives under different decisions (Table 2). 
The T-ONS model seeks to mitigate the disadvantages of a pure TLS display by providing dashboard displays of key variable of stakeholder concern and also a pressure gauge figure to indicate how close Traffic lights are to being triggered. Moreover, it provides extensive tables of outcomes of the preselected scenarios and the current realisation as a basis for more careful analysis by MCA or less formal decision support approaches. Finally, although the categorisation of TLS of the T-ONS model remains essentially subjective, this is less critical as its purpose is simply to communicate the stated positions of different stakeholder groups that were obtained, processed and utilised as previously described. These displays of the T-ONS model seem the easiest way to communicate the stakeholders' concerns to any decision support framework.

\subsection{The future of decision support}

Senior managers must themselves decide what decision support framework to adopt. In practice they will find, when using the 'N dimensional potato' approach in the North Sea that it does not currently seem possible to give a solution that will keep all the stakeholders in their comfort zones. If the main consideration of senior managers is to arrive as near as possible to a consensus solution then the T-ONS model and its stakeholder driven TLS should assist them directly. It may be easily used interactively to find the least uncomfortable solutions. Optimizations of favoured criteria that are constrained by stakeholder dislikes (using Excel's solver function) would also be a way to seek good solutions.

If alternatively senior managers wish to adhere to the teaching of Machiavelli7 that half measures (i.e. compromises) do not work then they may wish to directly adopt one of the pure strategy scenarios offered by the T-ONS model. If they cannot directly decide on a scenario they could seek the most suitable one using their own criteria and weighting in an MCA. In this latter case, the ' $\mathrm{N}$ dimensional potato' result will still be relevant as background on the likely reception to their management solution and will likely help them with deciding weightings.

The pilot implementation of the $\mathrm{N}$ dimensional potato approach in the North Sea case study exemplify its potential applications. Though the 'N dimensional potato' decision support framework was 'made for' decisions-makers 'at a decision level above' the stakeholders in the advisory councils, the feedback from stakeholders involved in the case study, as detailed in the result section, has led us to believe that it is an approach that could facilitate discussions on EBFM issues among stakeholders. The ' $\mathrm{N}$ dimensional potato' decision support approach would enable stakeholders to anticipate to a certain extent the reactions of other stakeholders to specific strategies/proposals.

Although stakeholders are probably already able to do this to a certain extent, the T-ONS model would also enable them to more easily explore the palatable parts of the fisheries potato for possible acceptable compromises in the spirit of cooperation and maybe even co-creation. The approach is also useful in improving management design so that the number of red lights can be minimized.

The 'N dimensional potato approach' clarifies values and facts for an EBFM decision without imposing normative considerations. The definition of the decision-space is based on a systematic gathering of stakeholders' preferences without considering the formal limitations of the current policy and legal frameworks. As a standard approach today efforts are focused on defining constrained optima by delineating the sustainability space within which stakeholders can explore options (Ballesteros et al., 2017); in contrast, the N-dimensional potato does not preclude any preference or pre-set 'the potato size', which is in itself an asset for the exploration of alternative policy options. Consequently, this approach is valuable for exploring such potential policy options.

\section{Acknowledgements}

The research leading to these results has received funding from the European Union's Seventh Framework Programme Project MareFrame: Co-creating Ecosystem-based Fisheries Management Solutions under Grant Agreement no. 613571. Particular thanks are due to Michael Andersen for suggesting the "N dimensional potato" idea and name. We remain grateful to the NSAC's Demersal Working Group for making time to discuss this at their meetings and for filling in the initial questionnaire and for providing feedback and co-creation support. The authors express a particular gratitude to all the members of the Pelagic Advisory Council and the North Sea Advisory Council for their active engagement and to their Executive secretariats for facilitating the process.

\section{References}

Ballesteros et al., 2017 M. Ballesteros, R. Chapela, P. Ramírez-Monsalve, J. Raakjaer, T.J. Hegland, K.N. Nielsen, U. Laksá and P. Degnbol, Do not shoot the messenger: ICES advice for an ecosystem approach to fisheries management in the European Union, ICES J. Mar. Sci. 75, 2017, 519-530, https://doi.org/10.1093/icesjms/fsx181.

Ballesteros, M., Chapela, R., Raakjaer, J., Ramirez-Monsalve, P., Hegland, T.J., Degnbol, P., Nielsen, K., Rahikainen, M., Baudron, A., Bartolino, V., Colloca, F., Ruiz, J., Rincón, M.M., Pope, J., Agnarsson, S., Elvarson, B. Stefansson, G., in prep. Who Joins the Table? A Critical Overview of the Co-Creation Approach for the Implementation of an Ecosystem-based Fisheries Management Approach.

Caddy J.F., A short review of precautionary reference points and some proposals for their use in data-poor situation, FAO FishTech. Paper 379, 1998, 1-29.

Caddy J.F., Limit reference points, traffic lights, and holistic approaches to fisheries management with minimal stock assessment input, Fish. Res. 56, 2002, 133-137.

Caddy J.F. and Mahon R., Reference points for fisheries management, FAO FishTech. Paper 347, 1995, 83.

Collie J.S., Gislason H. and Vinther M., Using AMOEBAs to display multispecies, multifleet fisheries advice, ICES J. Mar. SCi. 60, 2003, 709-720, https://doi.org/10.1016/S1054-3139(03)00042-0.

Cvitanovic C., Hobday A.J., van Kerkhoff L., Wilson S.K., Dobbs K. and Marshall N.A., Improving knowledge exchange among scientists and decision-makers to facilitate the adaptive governance of marine resources: a review of knowledge and research needs, Ocean Coast. Manag. 112, 2015, 25-35, https://doi.org/10.1016/j.ocecoaman.2015.05.002.

Dolan T.E., Patrick W.S. and Link J.S., Delineating the continuum of marine ecosystem-based management: a US fisheries reference point perspective, ICES J. Mar. Sci. 73, 2016, 1042-1050. 
EC, Regulation (EU) No 1380/2013 of the European Parliament and the Council of 11 December 2013 on the Common Fisheries Policy, amending Council Regulation (EC) No 1954/2003 and (EC) No 1224/2009 and replacing Council Regulation (EC) No 2371/2002 and (EC) No 639/2004 and Council Decision 2004/585/EC, Off. J. Eur. Union 2013, L 534/22-61 http://eur-lex.europa.eu/legal-content/EN/TXT/PDF/?uri=CELEX:32013R1380\&from=EN.

Foden J., Rogers S.I. and Jones A.P., A critical review of approaches to aquatic environmental assessment, Mar Pollut. Bull. 56, 2008, 1825-1833.

Garcia S.M., Precautionary approach to fisheries, FAO Fish. Tech. Paper 350 (2), 1995, 210.

Hall S.J. and Mainprize B., Towards ecosystem-based fisheries management, FishFish. 5, 2004, 1-20.

Hatchard J.L. and Gray T.S., From RACs to advisory councils: lessons from North Sea discourse for the 2014 reform of the European common fisheries policy, Mar. Pol. 47, 2014, 87-93.

Hilborn R., Pretty Good Ygood yield and exploited fishes, Mar. Pol. 34, 2010, 193-196.

ICES, Multispecies considerations for the North Sea stocks, ICES Advice 2013 vol. 6, 2013, June.

ICES, 2017. ICES, Mixed-fisheries advice for subarea 4, division 7.d, and subdivision 3.a.20 (North Sea, eastern English Channel, Skagerrak), ICES Advice on Fishing Opportunities, Catch, and Effort, 2017https://doi.org/10.17895/ ices.pub.3159, 30 June 2017.

Janssen R., On the use of multi-criteria analysis in environmental impact assessment in Tthe Netherlands, J. Multi-Crit Decis. Anal. 10, 2001, 101-109.

Kowalski K., Stagl S., Madlener R. and Omann I., Sustainable energy futures: methodological challenges in combining scenarios and participatory multi-criteria analysis, EurJ. Inf. Syst. 197, 2009, 1063-1074.

Larkin P.A., An epitaph for the concept of maximum sustained yield, Trans. Am. Fish. Soc. 106, 1977, 1-11.

Link J.S. and Browman H.I., Integrating what? Levels of marine ecosystem-based assessment and management, ICES J. Mar. Sci. 71, 2014, 1170-1173.

Mauser W., Klepper G., Rice M., Schmalzbauer B.S., Hackmann H., Leemans R. and Moore H., Transdisciplinary global change research: the co-creation of knowledge for sustainability, Current Opinion in Environmental Sustainability52013. Opin. Environ. Sustain. 5, 2013, 420-431.

Mustajoki J., Hämäläinen R.P. and Marttunen M., Participatory multicriteria decision analysis with Web-HIPRE: a case of lake regulation policy, Environ. Model. Softw. 19, 2004, 537-547.

Pope J., Fisheries resource management theory and practice, In: Taylor J.L. and Baird G.G., (Eds.), New Zealand Finfish Fisheries: The Resources and Their Management, 1983, New Zealand Trade Publications Limited; Auckland.

Pope, J.G., 2018. T-ONS: A Swift Transportable and User Friendly Multispecies Model of the North Sea That Describes the Main Trade-Offs Used in Decision Support in EBFM. This volume.

Pope, J.G., Weber, C.T., this issue. A Fabulous Attempt to Link the Cost of Compliance to EBFM.

Ramírez-Monsalve P., Raakjær J., Nielsen K.N., Laksá U., Danielsen R., Degnbol D., Ballesteros M. and Degnbol P., Institutional challenges for policy-making and fisheries advice to move to a full EAFM approach within the current governance structures for marine policies, Mar. Pol. 69, 2016a, 1-12.

Ramírez-Monsalve P., Raakjær J., Nielsen K.N., Santiago J.L., Ballesteros M., Laksá U. and Degnbol P., Ecosystem Approach to Fisheries Management (EAFM) in the EU - current science-policy-society interfaces and emerging requirements, Mar. Pol. 66,2016b, 83-92.

Sheppard S.R.J. and Meitner M., Using multi-criteria analysis and visualisation for sustainable forest management planning with stakeholder groups, For. Ecol. Manage. 207, 2005, 171-187.

Tress G., Tress B. and Fry G., Clarifying integrative research concepts in landscape ecology, Landsc. Ecol. 20, 2004, 479-493.

\section{Footnotes}

1 From St Augustine's Confessions (ca 397). Loosely translated: "Make me virtuous, God, but not yet".

2 As the song says: "You've got to accentuate the positive, eliminate the negative" (by Jonny Mercer, 1944).

3 Fmsy is the fishing mortality rate that achieves Maximum Sustainable Yield (MSY)

4 Available at: https://mareframe.github.io/dsf/dev/MCA2/DST.html?model=northSea.

5 For the landing obligation the European Commission has funded a specific research project (Discardless (www.discardless.eu). During the scoping exercises, both stakeholders and the research team explored the frame and focus of the research programme (EBFM),

facilitating the split between short-term acute needs in the stakeholders' agenda and the needs related to an effective implementation of an EBFM in the EU.

6 Using low cunning, by plotting a series of ones or zeros that indicate if the red (unacceptable level) is invoked at a criteria level. A similar series is plotted for the yellow (unappealing level of a criteria) and a series of ones for the green acceptable levels. Only the ones are plotted and the order is red over yellow over green such that the lights change as each worse level is reached by a criteria.

7 MachiavelliN. (1996) Discourses on Livy. Translated by Harvey C. Mansfield and Nathan Tarcov. Chicago: University of Chicago Press. 